\title{
Transmission curves and effective refraction indices of MKO near infrared consortium filters at cryogenic temperatures
}

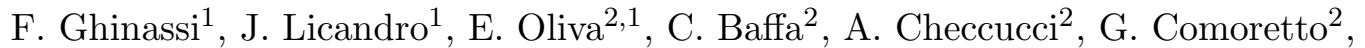 \\ S. Gennari ${ }^{2}$, and G. Marcucci ${ }^{3}$ \\ 1 Centro Galileo Galilei \& Telescopio Nazionale Galileo, PO Box 565, 38700 S. Cruz de La Palma, Spain \\ 2 Osservatorio Astrofisico di Arcetri, Largo E. Fermi 5, 50125 Firenze, Italy \\ 3 Università degli studi di Firenze, dipartimento di Astronomia, Largo E. Fermi 3, 50125 Firenze, Italy \\ Received 28 January 2002 / Accepted 19 February 2002

\begin{abstract}
We report transmission measurements at cryogenic temperatures for 4 broad-band filters of the Mauna Kea Observatories (MKO) near-infrared filter set and 5 narrow-band filters. The spectral scans were collected using the multi-mode IR instrument of the TNG telescope (NICS) in which these filters are permanently mounted and commonly used for astronomical observations. We determined the transmission curves at a temperature of $78 \mathrm{~K}$ and found no significant red-leak up to $2.6 \mu \mathrm{m}$, the data are available in electronic form on the TNG web page. We also estimated the variation of the wavelength response with the incidence angle and found it compatible with an effective refractive index of $\simeq 2$ (see Eq. (1)).
\end{abstract}

Key words. instrumentation: miscellaneous - instrumentation: spectrographs - infrared: general

\section{Introduction}

Near infrared imaging observations are usually made using a set of "standard" filters, the most popular being $J, H$, $K$ which match the 3 regions of clear atmospheric transmission at near infrared wavelengths, i.e. between 1.1 and $2.5 \mu \mathrm{m}$. Although these bands were first defined long ago (see Johnson 1962, 1964, 1968), no standard recipe exists for defining and manufacturing these filters. Consequently, different filters were used in different observatories and, in several cases, the filter transmission curve at the operating temperature of IR instruments $(\simeq 77 \mathrm{~K})$ is not available (see e.g. Moro \& Munari 2000).

Contrary to the broad-band filters at optical wavelengths, several of which can be manufactured using combinations of commercial coloured glasses, those for IR observations can only be produced by multi-layers coating. Although some firms have produced standard astronomical IR filters for many years, the stability of their characteristics is not always satisfactory. An instructive example is comparing the transmission curves of the standard $J$ filters manufactured by the same company for ESO and for the TNG (dotted and dashed curves in Fig. 1, respectively).

Clarifying this situation has been one of the main aims of the MKO near-infrared filter consortium (Simons \& Tokunaga 2002; Tokunaga et al. 2002). Thanks also to

Send offprint requests to: E. Oliva, e-mail: oliva@tng.iac.es the organizational effort of A. Tokunaga, a large number of observatories throughout the world could recently purchase a uniform set of carefully defined, high quality filters at an affordable price.

Since direct measurements of the transmission of these filters at cryogenic temperatures are scarce or, for some of them, lacking altogether, we report here accurate spectral scans at $78 \mathrm{~K}$ for the set of filters purchased for the multi-mode IR instrument of the Italian $3.5 \mathrm{~m}$ telescope (TNG). The broad-band filters were purchased as part of the first MKO filter consortium in 1998 that used OCLI as the filter vendor. The narrow-band filters were purchased as part of a separate filter consortium organized in 2000. We plan to repeat these measurements roughly every year to monitor the aging of the coating layers (if any), the results will be made available in the TNG web page (www.tng.iac.es/IRfilters.html) where one can also find the data presented here in electronic form.

\section{Measurements}

The data were collected in May 2001 using NICS, the near infrared camera and spectrometer which is permanently mounted on the Italian TNG telescope in La Palma. This instrument is a FOSC-type cryogenic focal reducer equipped with two interchangeable cameras feeding a Rockwell Hawaii $1024^{2}$ array. The camera used for the spectroscopic observations has a focal ratio of $\mathrm{F} / 4.3$ and 


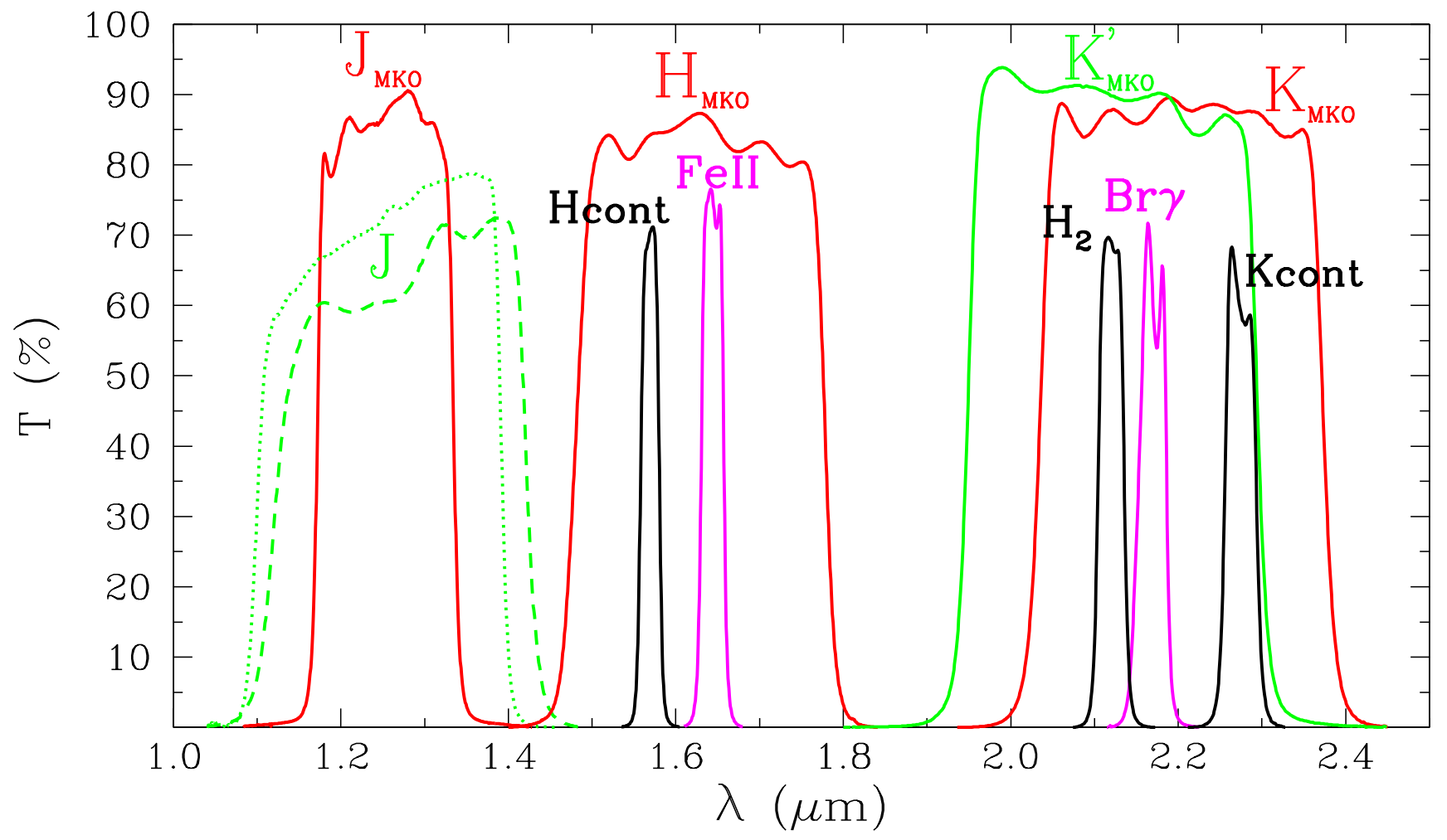

Fig. 1. Overview of the transmission curves of the filters of Mauna Kea Observatories (MKO) near infrared filter consortium. The data were taken at a temperature of $78 \mathrm{~K}$ and with the broad and narrow band filters illuminated at an incidence angle of $5^{\circ}$ and $3^{\circ}$, respectively. For comparison, we also include the curve relative to the "standard $J$ " filter of NICS measured in the same conditions (dashed line) as well as that of the "standard $J$ " filter used in the SOFI and ISAAC instruments of ESO (dotted line). The data for the ESO filter were downloaded from the ISAAC web page.

yields a sky projected scale of $0.25^{\prime \prime} /$ pixel. The spectroscopic modes are achieved by means of an Amici prism and a series of glass-resin grisms which can be inserted in the $\oslash 22 \mathrm{~mm}$ collimated beam (see e.g. Baffa et al. 2001).

All the grisms used for these measurements have their dedicated order sorter rigidly mounted inside the grism holder. Consequently, normal long-slit spectroscopic data are collected with the filter wheel in the "open" position. Therefore, by inserting a filter in the filter wheel, one can measure its transmission at the wavelengths covered by the disperser.

The measurements were performed as follows. A cold entrance slit of $0.095 \mathrm{~mm}$, with a projected size of 2 pixels onto the array, was illuminated by an halogen lamp through a diffuser. After the collimator, the light passed through a Lyot stop, the filter and grism wheels before reaching the camera and the detector. A first series of spectra of the halogen lamp were taken by inserting one of the dispersers available in the grism wheel followed, immediately after, by a series of spectra with a filter inserted in the filter wheel and, again, by measurements of the halogen lamp without any filter. Integration times were identical for all frames and long enough to obtain between 5000 and $30000 \mathrm{e}^{-}$per pixels at all the wavelengths of interest, i.e. a good level of illumination remaining well below the limits of non-linearity. Each of these cycles lasted a few minutes during which the input flux from the lamp was found to be stable within better than $1 \%$.

The dispersers used were the grisms $I J(0.89-1.45 \mu \mathrm{m}$, $5.5 \AA / \mathrm{pix}), J H(1.15-1.75 \mu \mathrm{m}, 6.6 \AA / \mathrm{pix}), J K^{\prime}(1.15-$ $2.20 \mu \mathrm{m}, 11.6 \AA / \mathrm{pix})$ and $H K(1.40-2.50 \mu \mathrm{m}, 11.2 \AA / \mathrm{pix})$ which allowed determining the filters transmission with quite fine spectral details. Measurements with a factor of about 10 lower dispersion, i.e. at $\lambda / \Delta \lambda \simeq 100$, were also taken with the Amici prism $(0.8-2.6 \mu \mathrm{m})$, these data were particularly useful to estimate the out-of-band blocking factor which, in the red part of the spectrum, and up to array cutoff wavelength of $2.6 \mu \mathrm{m}$, could be checked to a level of about $0.01 \%$.

The transmission of a given filter was simply determined by dividing frames taken with and without the filter in the collimated beam. Given the very high $\mathrm{s} / \mathrm{n}$ ratio of the spectra, the internal errors of the measurements are negligibly small. The actual accuracy of the curves is practically limited by systematic effects due, for example, to the fact that the filters are tilted by $3-5$ degrees relative to the optical axis and, when they are inserted in the beam, shift the pupil image by $\simeq 0.2 \mathrm{~mm}$, i.e. $\simeq 1 \%$ of the pupil diameter. Therefore, slightly different parts of the dispersers were illuminated in the measurements with and without the filter. Nevertheless, each filter transmission could be independently determined using at least two 
Table 1. Summary of filters characteristics ${ }^{1}$.

\begin{tabular}{llll}
\hline \hline Name & $\begin{array}{l}\text { cuton }^{2} \\
(\mu \mathrm{m})\end{array}$ & $\begin{array}{l}\text { cutoff }^{2} \\
(\mu \mathrm{m})\end{array}$ & $\begin{array}{l}<t>^{3} \\
(\%)\end{array}$ \\
\hline$J_{\mathrm{MKO}}$ & 1.172 & 1.335 & 84 \\
$H_{\mathrm{MKO}}$ & 1.481 & 1.779 & 81 \\
$K_{\mathrm{MKO}}^{\prime}$ & 1.949 & 2.295 & 88 \\
$K_{\mathrm{MKO}}$ & 2.035 & 2.374 & 85 \\
& & & \\
$\mathrm{Hcont}$ & 1.5580 & 1.5807 & 67 \\
FeII & 1.6304 & 1.6576 & 72 \\
$\mathrm{H} 2$ & 2.1061 & 2.1376 & 66 \\
$\mathrm{Br} \gamma^{4}$ & 2.1515 & 2.1868 & 62 \\
$\mathrm{Kcont}^{4}$ & 2.2551 & 2.2941 & 60 \\
\hline
\end{tabular}

1 Measured at $T=78 \mathrm{~K}$ and at an incidence angle of $5^{\circ}$ (broad band filters) and $3^{\circ}$ (narrow band filters).

2 Half-power points.

3 Average transmission between $75 \%$ points.

4 The transmission curve is quite irregular, see Fig. 2.

different dispersers and, consequently, we could estimate that the systematic errors are $<4 \%$ in the absolute values and $\leq 1 \%$ in the shape of the curves.

Wavelength calibration was performed using exposures of Ar and Xe lamps which were taken before/after every change of disperser, the dispersion was always found to be stable within $<0.1$ pixels, i.e. a factor of $>20$ better than the $\lambda / \Delta \lambda \simeq 1000$ resolving power of the spectra.

The variation of the transmission curves with incidence angle was determined by extracting spectra at different distances for the array center. The data for narrow band filters spanned a range of incidence angle from $3^{\circ}$ to $6.5^{\circ}$ while those for broad band filters extended between $5^{\circ}$ and $7.6^{\circ}$.

\section{Results}

The measured transmissions of the MKO near-infrared consortium filters mounted in NICS are displayed in Fig. 1 which also includes, for comparison, the curves relative to the standard $J$ filter (see the caption for details). The corresponding half-power points and average transmissions are summarized in Table 1.

Figure 2 is a zoom on the transmission curves of the narrow band filters and includes measurements taken at different incidence angles. The shift of the central wavelength and half power points can be well represented by the relationship

$\lambda(\theta) \simeq \lambda\left(0^{\circ}\right) \cdot \sqrt{1-\frac{\sin ^{2} \theta}{n_{\mathrm{eff}}^{2}}}$

where $n_{\text {eff }}$ is the effective refraction index. Within the range of angle spanned by our measurements, all the data are compatible with $n_{\text {eff }} \simeq 2$, a quite typical value for IR interference filters (see e.g. Vanzi et al. 1998).

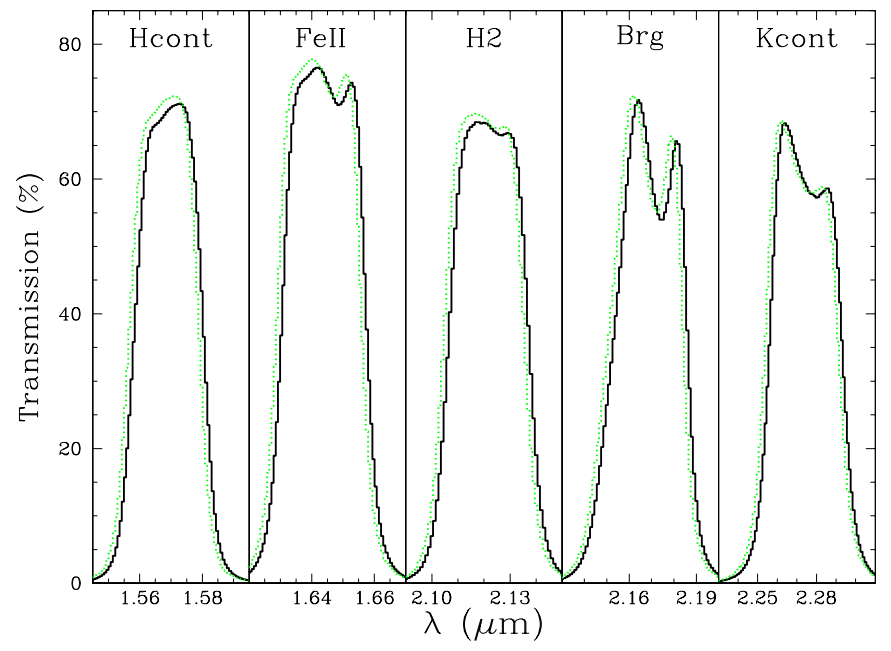

Fig. 2. Detailed view of the narrow band filter curves. The solid line is for an incidence angle of $3^{\circ}$ while the dashed curve refer to measurements at $6.5^{\circ}$. The shift in wavelength is compatible with an effective refractive index of 2 for all filters.

\section{Conclusions}

This paper reports measurements of the spectral transmission at $78 \mathrm{~K}$ of 4 broad-band filters of the Mauna Kea Observatories (MKO) near-infrared filter set and 5 additional narrow-band filters. The measured curves confirm the good quality of most of the filters and could be useful to all those observatories who are, or will be using the same filters. The transmission curves are also available in electronic form on the TNG web pages (www.tng.iac.es/IRfilters.html). We plan to repeat these measurements roughly every year to monitor the aging of the coating layers (if any), the results will be made available on the same web pages.

Acknowledgements. We are grateful to Alan Tokunaga for all his work and efforts in organizing the consortium and taking care of the contacts with the manufacturing companies.

This paper is based on observations made with the Italian Telescopio Nazionale Galileo (TNG) operated on the island of La Palma by the Centro Galileo Galilei of the CNAA (Consorzio Nazionale per l'Astronomia e l'Astrofisica) at the Spanish Observatorio del Roque de los Muchachos of the Instituto de Astrofisica de Canarias.

\section{References}

Baffa, C., Comoretto, G., Gennari, S., et al. 2001, A\&A, 378, 722

Johnson, H. L. 1962, ApJ, 135, 69

Johnson, H. L. 1964, Bull. Tonantzintla Tacubaya Obs. 3, 305

Johnson, H. L. 1968, ApJ, 152, 465

Moro, D., \& Munari, U. 2000, A\&AS, 147, 361

Simons, D. A., \& Tokunaga, A. T. 2002, PASP, in press [astro-ph/0110594]

Tokunaga, A. T., Simons, D. A., \& Vacca, W. D. 2002, PASP, in press [astro-ph/0110593]

Vanzi, L., Gennari, S., Ciofini, M., \& Testi, L. 1998, Exp. Astr., 8,177 